\title{
Estudos brasileiros sobre nefrologia nas teses e dissertações de enfermagem
}

\author{
Brazilian studies on nephrology produced in nursing thesis and dissertations \\ Estudios brasileros sobre nefrología en las tesis y disertaciones de enfermería
}

\section{Glória Maria Custódio de Carvalho', Francisca Elisângela Teixeira Lima", Islene Victor Barbosa', Elisabeth Mesquita Melo'}

'Universidade de Fortaleza. Departamento de Enfermagem. Fortaleza, CE

"Universidade Federal do Ceará, Departamento de Enfermagem. Fortaleza, CE

Submissão: 01/07/2009

Aprovação: 12/09/2010

\section{RESUMO}

O objetivo foi avaliar a produção científica na enfermagem brasileira sobre nefrologia nas teses e dissertações publicadas de 2001 a 2007. Foi realizado um estudo bibliométrico apartir dos teses e dissertações de enfermagem catalogadas no Centro de Estudos e Pesquisas em Enfermagem. Detectou-se 50 trabalhos sobre nefrologia, predominando: 45 (90\%) dissertações; 30 (60\%) no estado de São Paulo; 18 (36\%) em clínicas de diálise; 39 (78\%) sujeitos foram pacientes. Quanto aos tipos de estudos predominaram: 26 (52\%) exploratórios, 28 (56\%) levantamento e 27 (54\%) Quantitativos. De acordo com o tema, a hemodiálise teve maior destąue, com 21 (42\%) publicações. As pesquisas científicas voltadas para essa temática possibilitam uma assistência multi e interdisciplinar, de forma individualizada, sistematizada e humanizada a essa clientela.

Descritores: Unidades hospitalares de hemodiálise; Diálise peritoneal; Nefropatias; Enfermagem.

\section{ABSTRACT}

The purpose was to evaluate the scientific production in Brazilian nursing about nephrology in theses and dissertations published from 200 I to 2007. A bibliometric study was carried out with the nursing tesis and dissertations according to the Center of Studies and Researches in Nursing catalog. It was found 50 works on nephrology, predominating: 45 (90\%) dissertations; 30 (60\%) in the state of São Paulo; 18 (36\%) in clinics of dialysis; 39 (78\%) samples were patients. Considering the types of study, it predominated: 26 (52\%) exploratory, 28 (56\%) survey and 27 (54\%) Quantitative. Concerning the theme, hemodialysis predominated with 21 (42\%) publications. Scientific researches on this theme make possible a multi and interdisciplinary assistance to these clients, in an individualized, systematized and humanized way.

Key words: Hemodialysis units, hospital; Peritoneal dialysis; Kidney diseases; Nursing.

\section{RESUMEN}

El objetivo fue evaluar la producción científica en la enfermería brasileña sobre nefrología en las tesis y disertaciones publicadas de 2001 a 2007. Es un estudio bibliometrico, realizado con las tesis y desertaciones de enfermería catalogadas en el Centro de Estudios e Investigaciones en Enfermería. Se detectaron 50 trabajos sobre de nefrología, predominando: 45 (90\%) disertaciones; 30 (60\%) en el Estado de São Paulo; 18 (36\%) en clínicas de diálisis; 39 (78\%) la muestra fueron los pacientes. Los tipos de estudios predominaron: 26 (52\%) exploratorios, 28 (56\%) levantamiento y 27 (54\%) cuantitativos. De acuerdo con el tema, la hemodiálisis tuvo mayor destaque, con 21 (42\%) publicaciones. Las investigaciones científicas dirigidas a esta temática posibilitan una asistencia multi e interdisciplinar, de forma individualizada, sistematizada y humanizada a estos pacientes.

Palabras-clave: Unidades de hemodiálisis en hospital; Diálisis peritoneal; Enfermedades renales; Enfermería.

\footnotetext{
AUTOR CORRESPONDENTE Francisca Elisângela Teixeira Lima. Avenida dos Expedicionários, 3406, apto I203. CEP 604I0-4I0. Fortaleza, CE.

E-mail: felisangela@yahoo.com.br
} 


\section{INTRODUÇÃO}

A insuficiência renal crônica (IRC) é uma doença de alta morbimortalidade, sua incidência e prevalência continuam aumentando progressivamente no Brasil e em todo o mundo. Aproximadamente 20 a 30 pacientes apresentam algum grau de disfunção renal para cada paciente Que necessita de tratamento dialítico(I).

No Brasil, no ano de 2000, havia 42.695 pacientes Que realizavam tratamento dialítico, cujo número, em 2009, Quase duplicou para 77.589 pacientes. Contudo, há aproximadamente um milhão de pessoas com doença renal, mas 70\% destes são sub-diagnosticados. Estima-se Que para cada paciente em diálise existem de 15 a 30 pacientes Que possuem alguma doença renal crônica ${ }^{(2)}$.

A enfermagem tem estruturado princípios, normas, significados e formulado um corpo de conhecimentos próprio, Que proporcione base sólida ao desenvolvimento da prática ${ }^{(3)}$. Para tanto, os profissionais enfermeiros vem realizando pesquisas que buscam proporcionar uma melhoria do cuidado, resultando em uma melhor Qualidade de vida para o paciente.

O contexto descrito levou-nos ao objetivo geral: avaliar a produção científica na enfermagem brasileira sobre a temática nefrologia nas teses e dissertações publicadas no período de 2001 a 2007. E como específicos: averiguar os aspectos teóricometodológicos utilizados no desenvolvimento das dissertações e teses da enfermagem brasileira; e identificar Quais as temáticas da nefrologia Que o enfermeiro pesquisou nas dissertações e teses publicadas no período de 2001 a 2007.

\section{MÉTODO}

Tratou-se de um estudo bibliométrico Que visou conhecer a produção científica contida nas teses e dissertações de enfermagem produzidas no Brasil, em programas de pós-graduação strictu sensu sobre a temática nefrologia no período de 2001 a 2007, traçando assim um perfil destas publicações no período considerado.

Os materiais utilizados para o desenvolvimento da pesquisa estavam disponíveis no site da Associação Brasileira de Enfermagem (ABEn), (http:// www.abennacional.org.br), na área do Centro de Estudos e Pesquisas em Enfermagem (CEPEn), no catálogo de teses e dissertações de enfermagem, desenvolvidas em todos os programas de pósgraduação em enfermagem do Brasil, reconhecidas pela Coordenação de Aperfeiçoamento de Pessoal de Nível Superior (CAPES), no período de 2001 a 2007. Esse período foi selecionado tendo em vista Que o site disponibilizava os documentos referentes a esses anos no período da coleta de dados.

A coleta de dados ocorreu entre os meses de setembro a dezembro de 2008. Para a seleção das teses e dissertações acerca da nefrologia, buscou-se pelos Descritores em Ciências da Saúde (DECS), cadastrados na Biblioteca Virtual em Saúde, os quais foram: insuficiência renal aguda, insuficiência renal crônica, diálise renal, diálise peritoneal, transplante de rim e nefropatias. E para complementar a busca utilizou-se a palavra-chave: hemodiálise.

Para avaliar os aspectos metodológicos utilizados nos estudos desenvolvidos sobre nefrologia, utilizou-se a classificação das peseuisas com base nos objetivos, nos delineamentos e na natureza do estudo ${ }^{(4-6)}$.

\section{RESULTADOS}

$\mathrm{Na}$ busca pelos estudos desenvolvidos nos mestrados e doutorados brasileiros na pós-graduação em enfermagem, detectouse 50 trabalhos acerca da nefrologia, no período de 2001 a 2007. Dentre os Quais, 45 (90\%) são dissertações desenvolvidas no mestrado e apenas 5 (10\%) são teses referentes à conclusão do doutorado.

Os catálogos do CEPEn pesQuisados são referentes ao período de 2001 a 2007, entretanto há trabalhos concluídos em 2000 Que só foram publicados no ano seguinte. Portanto, considerou-se neste estudo o ano de conclusão do mestrado ou doutorado (Figura I).

Contatou-se que o ano de 2001 houve um predomínio de produção científica acerca da nefrologia, totalizando 10 (20\%) trabalhos. Seguido de 2005 e 2007 Que igualmente produziram sete (14\%) trabalhos; e em 2000, 2003 e 2004, houve a produção de seis (12\%) trabalhos por ano. Por último encontra-se 2002 e 2006 com apenas Quatro (8\%) produções.

Ao analisarmos a origem da produção avaliada, percebe-se nitidamente a predominância de estudos desenvolvidos na Região Sudeste. Dentre os estudos realizados, 4 I (82\%) foram desenvolvidos nesta região, contrastando com apenas Quatro (8\%) desenvolvidos na Região Nordeste.

Entre os estudos desenvolvidos na Região Sudeste, 14 foram na Universidade de São Paulo (USP), nove na USP de Ribeirão Preto, cinco na Universidade Federal de São Paulo (UNIFESP), Quatro na Universidade do Rio de Janeiro (URJ), dois na Universidade Federal do Rio de Janeiro (UFRJ), Quatro na Universidade Federal de Minas Gerais (UFMG) e dois na Universidade Estadual de Campinas (UNICAMP).

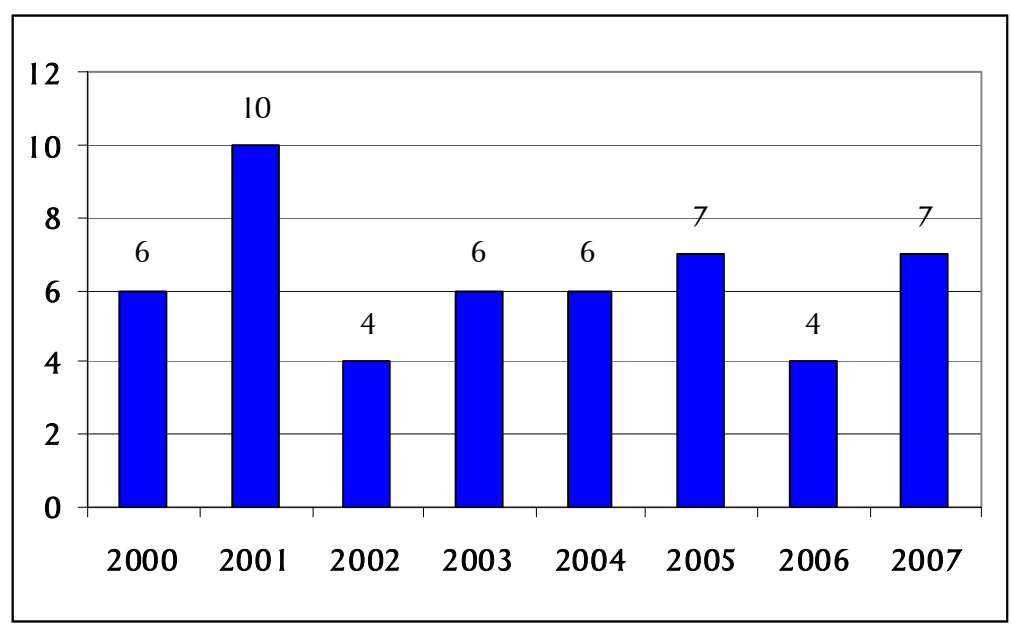

Figura 1. Caracterização dos resumos publicados nos catálogos do CEPEn de 2001 - 2007, por ano do estudo. 
Na Região Nordeste, apenas a Universidade Federal do Ceará (UFC) e a Universidade Federal da Bahia (UFBA) publicaram os estudos desenvolvidos no período considerado, sendo três na UFC e um na UFBA.

Dentre as outras instituições de ensino superior citadas estão: Fundação Oswaldo Cruz (1), Universidade Federal do Paraná (1), Universidade de Brasília (2), Universidade Federal de Santa Catarina (2). A região sul destaca-se pelo reduzido número de publicações, apenas 3 no período considerado.

Entre os trabalhos publicados no período considerado 1 I autores não especificaram no resumo o local em Que foi realizada a coleta de dados. Entre os Que citaram, a maioria das coletas ocorreu em unidades de saúde, sendo 37 no total, predominando as clínicas de diálise (18), seguida pelos hospitais (14) e ambulatórios (2), estudos experimentais em laboratórios (3). Apenas duas pesquisas foram desenvolvidas em domicílios de pacientes.

Em relação aos sujeitos envolvidos na pesQuisa, 39 abordaram os pacientes, representando o maior percentual (78\%), os profissionais foram investigados em apenas Quatro trabalhos, igualmente aos familiares e três experimentais com animais cobaias de laboratório.

Entre os 39 pacientes estudados, 15 eram adultos, oito idosos, dois adolescentes, uma criança e 13 foram desenvolvidos com o paciente, mas não foi especificada a faixa etária. Dos Quatro estudos desenvolvidos com profissionais, três trabalharam somente com o enfermeiro e um estudo com enfermeiros e médicos. Os estudos experimentais realizaram as seguintes investigações: a ação da isoflavona e o sistema heme-oxigenase na insuficiência renal aguda isQuêmica em ratos, o papel da heme-oxigenase na proteção pelas estatinas na insuficiência renal aguda isQuêmica em ratos e Lesão renal aguda por glicerol: efeito antioxidante da Vitis vinifera $L$.

Quanto aos tipos de estudos, 26 (52\%) foram exploratórios, 22 (44\%) descritivos e dois (4\%) explicativos. Quanto ao delineamento houve: 28 (56\%) estudos de levantamento, nove (18\%) experimentais, cinco (10\%) documentais, cinco (10\%) pesQuisa-ação e três (6\%) estudos de caso. E em relação à natureza do estudo, 27 (54\%) foram Quantitativos e 23 (46\%) Qualitativos.

Com relação ao referencial teórico, 42 (84\%) estudos apresentavam claramente seu referencial teórico, dentre eles destacam-se: 22 (44\%) trabalhos positivistas; 12 (24\%) foram classificados como outros e divididos da seguinte forma: Meihy (2), Sócio humanística ( I ), História oral de vida proposta por Bury

\begin{tabular}{|lc|}
\hline TEMA & n \\
\hline Hemodiálise & 2 l \\
Insuficiência renal crônica & 13 \\
Cuidado ao paciente renal & 11 \\
Qualidade de vida & 8 \\
Complicações de acesso à diálise & 6 \\
Diálise peritoneal & 4 \\
Unidade de Terapia Intensiva & 3 \\
Transplante renal & 3 \\
Insuficiência renal aguda & 3 \\
Promoção da saúde & 2 \\
Diálise e suas complicações & 2 \\
Outros & 6 \\
\hline
\end{tabular}

Quadro 1. Temas abordados nos estudos.
(1), Jean Watson(1), Mayeroff (1), Minayo (1), Imogene King (1), Materialismo histórico e dialético (1), Enfermagem familiar na promoção da saúde (I), Modelo teórico, Trajetória da Doença Crônica, Que se fundamenta em três processos: ruptura biográfica, impacto do tratamento, adaptação e manejo da doença (1), Roy (1). Os demais estudos foram: cinco fenomenológicos e três etnográficos. Após a análise de todos os resumos percebemos Que oito trabalhos não basearam suas pesQuisas em nenhum referencial teórico.

Após analisar os resumos publicados percebe-se Que o tema mais abordado foi a hemodiálise, aparecendo em 2 I (42\%) entre teses ou dissertações estudadas, seguido pela insuficiência renal crônica Que foi discutida em 13 (26\%) dos trabalhos publicados; e o cuidado ao paciente renal apareceu em I I (22\%) publicações do período considerado.

De acordo com o Quadro I observa-se Que 6 (12\%) trabalhos foram classificados como outros, os temas abordados foram os seguintes: fatores de risco para IRA após revascularização do miocárdio, sobrevida do paciente renal em diálise e gastos, diagnósticos de enfermagem, nefropatia diabética, humanização e tratamento conservador. Cada tema foi abordado em apenas um (2\%) estudo publicado.

\section{DISCUSSÃO}

A enfermagem, ao longo das últimas décadas, tem se empenhado na construção de um corpo de conhecimento marcado por conceitos amplos, focalizando a sistematização da assistência ${ }^{(7-}$ ${ }^{8)}$. Isso tem sido intensificado nos programas de pós-graduação em enfermagem, nível mestrado e doutorado.

Em relação à construção do conhecimento sobre nefrologia, foram detectados 50 trabalhos desenvolvidos na pós-graduação brasileira de enfermagem, com predomínio de 45 dissertações, podendo ser decorrente do maior Quantitativo de mestrados no Brasil. Conforme exposto CAPES há 35 Programas de pósgraduação no Brasil, com 49 cursos distribuídos da seguinte forma: 32 mestrados acadêmicos, dois mestrados profissionalizantes e 15 doutorados ${ }^{(9)}$.

Diante da análise do período de publicação, observa-se Que os trabalhos produzidos nos anos de 2000 e 200 I somam 16 (32\%), encuanto nos anos 2006 e 2007 juntos realizaram apenas 1 I (22\%) trabalhos. Preocupa-se com essa redução, pois, sabe-se Que há uma necessidade cada vez maior de se pesQuisar sobre a temática, devido as suas peculiaridades, pois essa clientela requer uma assistência cada vez mais sistematizada, individualizada e humanizada. Além disso, vem ocorrendo um aumento substancial de pessoas em tratamento dialítico, passando de 42.695 pacientes em 2000 para 77.589 em $2009^{(2)}$.

O predomínio da Região Sudeste pode ser decorrente da maioria dos cursos de pós-graduação estar localizada nessa região do país, podendo influenciar diretamente nesses resultados. Nos últimos anos, uma política de incentivo à pesQuisa tem se estabelecido nas outras regiões do Brasil, na tentativa de disseminar a pós-graduação em enfermagem nas cinco regiões brasileiras.

O processo da construção do conhecimento reflete as transformações do movimento histórico por Que passa a enfermagem. Estas transformações parecem evidenciar o movimento dinâmico entre 
ressonâncias e dissonâncias, na construção do conhecimento, com vistas a um avanço criador nas práticas sócio/política (ensino, pesquisa e assistência) de enfermagem ${ }^{(10)}$.

Nesse processo de construção do conhecimento os enfermeiros vem buscando utilizar diferentes tidos de estudo. Contudo, predominaram os estudos exploratórios e descritivos, enQuanto os estudos explicativos foram pouco desenvolvidos. O delineamento predominante foi o levantamento (56\%), o Qual se caracteriza pela interrogação direta das pessoas cujo comportamento se deseja conhecer, além de ter como vantagens: conhecimento direto da realidade; economia e rapidez; e Quantificação ${ }^{(1)}$. Contudo, apenas $18 \%$ foram experimentais, sendo considerado um número reduzido, talvez pela dificuldade de acesso dos pesquisadores enfermeiros aos laboratórios de ciências básicas e ao baixo investimento nessas pesquisas pelos programas de Pós-Graduação em Enfermagem.

Quanto à natureza dos estudos, percebe-se Que na enfermagem há um predomínio de estudos Quantitativos (54\%), os Quais derivam de amostras relativamente grandes ou representativas Que trazem muitos elementos positivos, pois devem ter poder de generalização, precisão, controle de variáveis estranhas, fidedignidade e mensuração(1) ${ }^{(1)}$ Contudo, também há uma Quantidade significativa de estudos Qualitativos (46\%), as Quais são essências para melhoria da prática assistencial da enfermagem, visto Que direcionam a uma experiência social, valem-se da fonte oral e se encaminham na busca dos significados de vivências para os sujeitos ${ }^{(12)}$.

Constata-se Que essa aproximação da enfermagem com as ciências humanas é justificada pelo seu direcionamento ao cuidar de seres humanos, dos Quais necessita conhecer as nuanças e subjetividades a fim de traçar linhas de ação para o cuidar.

Em relação ao tema relacionado à área de nefrologia, destacase a hemodiálise, tendo em vista Que é a terapia mais conhecida e aplicada a clientela com insuficiência renal. lá a diálise peritoneal, tratamento domiciliar realizado pelo próprio paciente ou cuidador devidamente treinados, foi abordada em poucos trabalhos. Esses achados devem ser decorrentes do elevado número de enfermeiros Que trabalha em hemodiálise em relação ao Quantitativo de enfermeiros que atuam em diálise peritoneal, bem como a clientela submetida a tais tratamentos.

\section{CONCLUSÃO}

Após avaliar os 50 estudos desenvolvidos acerca da nefrologia na pós-graduação strictu sensu brasileira no período de 2001 a 2007, constatou-se Que os aspectos teórico-metodológicos predominantes nos foram: dissertações (90\%), realizados no estado de São Paulo (60\%); em clínicas de diálise (36\%); cujos sujeitos do estudo foram os pacientes (78\%). Quanto aos tipos de estudos predominaram: exploratório (52\%), levantamento (56\%) e Quantitativo (54\%).

E as temáticas foram bastante diversificadas, contemplando a insuficiência renal aguda e crônica, as formas de tratamento substitutivo, assistência de enfermagem, Qualidade de vida, promoção da saúde, dentre outros.

Isso remete as peculiaridades do paciente com insuficiência renal, cabendo aos profissionais da saúde aprofundar e construir os conhecimentos acerca dessa clientela, mediante novas pesquisas científicas acerca dos temas já abordados, bem como de temas que merecem maior fundamentação científica para transformação e melhoria da prática assistencial.

Então, percebe-se a importância de pesQuisas científicas voltadas para essa temática, possibilitando uma assistência multi e interdisciplinar, de forma holística, individualizada, sistematizada e humanizada a essa clientela, enfatizando a cientificidade e segurança do cuidado prestado.

\section{REFERÊNCIAS}

1. Pinho FMO, Sodré EM, Silva IO. Insuficiência Renal Crônica. Tratamento Conservador e Dialítico. Ars Cvrandi: Clín Méd 2003; 38(6): 50-6.

2. Sociedade Brasileira de Nefrologia. Censo 2009. [citado em 2010 Maio 26]. Disponível em: http://www.sbn.org.br/pdf/ censo_SBN_2009_final.pdf

3. Moreira TMM, Jorge MSB; Lima FET. Análise das dissertações e teses de enfermagem sobre adolescência, Brasil, 1979-2000. Rev Bras Enferm 2004; 57(2): 217-22.

4. Gil AC. Como Elaborar Projetos de pesquisa. São Paulo: Atlas; 2002.

5. Lakatos EM, Marconi MA. Metodologia do trabalho científico. São Paulo: Atlas; 200I.

6. Creswell IW. Projeto de Pesquisa Métodos Qualitativo, Quantitativo e Misto. Porto Alegre: Artmed; 2007.

7. França ISX, Farias FSAB, Sobreira TT, Fraga MNO, Damasceno MMC. Análise de dissertações de mestrado em enfermagem à

luz da bioética. Rev Bras Enferm 2002; 55(5): 495-502.

8. Carvalho V. Tendências da pesquisa na enfermagem. In: Anais do $2^{\circ}$ Fórum Mineiro de Enfermagem; 2000; set 18-21; Uberlândia, Brasil. Uberlândia: Universidade Federal de Uberlândia; 2000. p.55-68.

9. Coordenação de Aperfeiçoamento de Pessoal de Nível Superior. [citado em 2009 Jul 16]. Disponível em: http:// www.capes.gov.br/

10. Padilha MICS, Silva AL, Borenstein MS. Os congressos brasileiros - pontes para a liberdade e transformação da enfermagem. Rev Latino-Am Enfermagem 2001; 9(3): 7-13.

11. Polit DF, Beck CT, Hungler BP. Fundamentos de pesquisa em enfermagem. Métodos, avaliação e utilização. Porto Alegre: Artmed; 2004.

12. Martinelli ML. Pesquisa Qualitativa: um instigante desafio. São Paulo: Veras editora; 2003. 